\title{
ArcheoSciences
}

Revue d'archéométrie

$40 \mid 2016$

Varia

\section{Datation archéomagnétique de deux fours du haut Moyen-Âge à Cumes (Campanie, Italie)}

Archaeomagnetic dating of two Early Middle Age kilns at Cumae (Campania, Italy)

Gwenael Hervé, Priscilla Munzi-Santoriello, Philippe Lanos, Jean-Pierre Brun, Paolo Caputo et Cristina Regis

\section{OpenEdition}

\section{Journals}

Édition électronique

URL : https://journals.openedition.org/archeosciences/4719

DOI : 10.4000/archeosciences.4719

ISBN : 978-2-7535-5384-2

ISSN : 2104-3728

Éditeur

Presses universitaires de Rennes

Édition imprimée

Date de publication : 30 décembre 2016

Pagination : $35-45$

ISBN : 978-2-7535-5382-8

ISSN : 1960-1360

\section{Référence électronique}

Gwenael Hervé, Priscilla Munzi-Santoriello, Philippe Lanos, Jean-Pierre Brun, Paolo Caputo et Cristina Regis, « Datation archéomagnétique de deux fours du haut Moyen-Âge à Cumes (Campanie, Italie) », ArcheoSciences [En ligne], 40 | 2016, mis en ligne le 30 décembre 2018, consulté le 24 février 2022. URL : http://journals.openedition.org/archeosciences/4719 ; DOI : https://doi.org/10.4000/ archeosciences.4719 


\title{
Datation archéomagnétique de deux fours du haut Moyen Âge à Cumes (Campanie, Italie)
}

\section{Archaeomagnetic Dating of Two Early Middle Age Kilns at Cumae (Campania, Italy)}

\author{
Gwenaël Hervéa, c, d, Priscilla Munzi-Santoriello ${ }^{\text {b }}$ Philippe Lanos ${ }^{c, d}$, \\ Jean-Pierre Brun ${ }^{\mathrm{e}}$, Paolo Caputo ${ }^{\dagger f}$ et Cristina Regis ${ }^{g}$
}

\begin{abstract}
Résumé : La datation archéomagnétique porte sur deux fours découverts dans la ville basse de Cumes, un four de tuilier retrouvé dans la nécropole de la Porte médiane et un four de potier retrouvé près de la Cava greca. Les deux fours sont datés par la stratigraphie et la typochronologie entre le ve et le VII siècle apr. J.-C. Les échantillons, prélevés par carottage dans les briques des parois des fours, ont été analysés par désaimantation thermique et par le protocole de Thellier-Thellier. Les deux fours donnent de très bons résultats d'archéointensité et présentent un très bon regroupement des directions d'aimantation. La différence significative entre les deux archéointensités moyennes indique que la fin du fonctionnement des deux fours n'est pas contemporaine. La datation archéomagnétique a été réalisée avec les prédictions à Cumes du modèle géomagnétique européen SCHA.DIF.3k. Le four de la Cava greca est daté entre 590 et 640 apr. J.-C. à $95 \%$ de confiance. Au même niveau de confiance, le four FR59006 de la nécropole de la Porte médiane est daté entre 540 et 645 apr. J.-C. La seconde moitié du vie siècle apparaît la période la plus probable. Ces deux datations archéomagnétiques confortent et précisent les datations archéologiques. Nos résultats placent le fonctionnement des deux secteurs artisanaux à l'époque byzantine et documentent les réappropriations médiévales de la ville antique.
\end{abstract}

\begin{abstract}
The archaeomagnetic study focuses on two kilns, unearthed in the lower city of Cumae, a tile kiln in the necropolis of the Median Gate and a

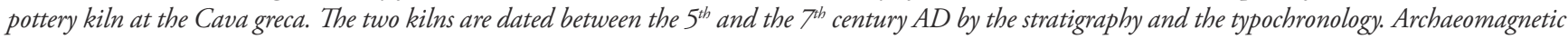
samples, cored in the bricks of the kiln walls, were analyzed by thermal demagnetization and by the Thellier-Thellier protocol. The two kilns provide very good archaeointensity results and the directions of the thermoremanent magnetization are well grouped. The significant difference between the two mean archaeointensities indicates that the last use of the two kilns were not contemporaneous. Archaeomagnetic dating is performed with the predictions at Cumae of the European model of the geomagnetic field SCHA.DIF.3k. The kiln from the Cava greca is dated between 590 and 640 AD at 95 per cent of confidence. At the same confidence level, the kiln FR59006 from the necropolis of the "Median Gate" is dated between 540 and 645 AD. The second half of the $\sigma^{\text {th }}$ century appears the most probable dating. These two archaeomagnetic datings concur and precise the archaeological dating. Our results date the use of the workshops in the Byzantine period and document the medieval changes of the antique city.
\end{abstract}

Mots clés : archéomagnétisme, datation, four de potier, four de tuilier, haut Moyen Âge, Italie.

Keywords: archaeomagnetism, dating, pottery kiln, tile kiln, Early Middle Age, Italy.

\footnotetext{
a Department für Geo- und Umweltwissenschaften, Ludwig-Maximilians Universität, Theresienstrasse 41, 80333 MÜNCHEN, Allemagne. (gwenaelherve. amag@gmail.com)

${ }^{b}$ Centre Jean Bérard, USR 3133 CNRS - EfR, Via Crispi 86, 80121 NAPOLI, Italie. (priscilla.munzi@cnrs.fr)

'Centre de Recherches en Physique Appliquée à l'Archéologie, UMR 5060 IRAMAT-CRPAA, esplanade des Antilles, 33607 PESSAC cedex, France.

' Géosciences Rennes, UMR 6118, Université de Rennes 1, Campus de Beaulieu, 35042 RENNES cedex, France. (philippe.lanos@univ-rennes1.fr)

- Collège de France, 11 place Marcelin-Berthelot, 75005 PARIS, France. (jean-pierre.brun@college-de-france.fr)

${ }^{f}$ Soprintendenza Archeologia Campania, Piazza Museo Nazionale 19, 80135 NAPOLI, Italie.

${ }^{g}$ Archéologue indépendante. (cristinaregis@libero.it)
} 


\section{INTRODUCTION}

La ville de Cumes, située au nord-ouest de la baie de Naples $\left(40,850^{\circ} \mathrm{N}, 14,055^{\circ} \mathrm{E}\right)$ en face de l'île d'Ischia, est une des premières colonies grecques en Italie du sud avec une fondation par les Eubéens vers 730 av. J.-C. La cité devient samnite à la fin du v siècle av. J.-C. puis romaine à partir des dernières décennies du Iv siècle av. J.-C. Elle s'organisait alors en une ville haute, l'Acropole, où étaient localisés les principaux temples, et une ville basse concentrant les édifices publics et l'habitat. L'Antiquité tardive est une période troublée avec notamment les guerres entre les Goths et l'Empire byzantin, qui se concluent par la conquête de la Campanie par les Byzantins en 553 apr. J.-C. L'occupation du site est documentée jusqu'aux premières décennies du XIII ${ }^{e}$ siècle.

L'Antiquité tardive et l'époque byzantine, au cours desquelles la ville a connu de nombreuses transformations, restent des périodes encore peu connues. Un castrum a été érigé sur l'Acropole. La ville basse a également été touchée par cette réaffectation des espaces qui reflète d'importants changements historiques et matériels. L'installation d'espaces artisanaux a notamment été mise en évidence dans la nécropole romaine de la Porte médiane et près de la Cava greca (figure 1), où ont respectivement été mis au jour deux fours de tuiliers et un four de potier. Ces structures sont datées par la stratigraphie et le mobilier archéologique associé entre le $\mathrm{v}^{\mathrm{e}}$ et le $\mathrm{VII}^{\mathrm{e}}$ siècles apr. J.-C. Afin de préciser la datation de ces espaces artisanaux et des fours, une analyse archéomagnétique a été menée en 2012-2013 sur deux structures, le four FR59006 découvert dans la nécropole de la Porte Médiane et le four mis au jour près de la Cava greca. Cet article présente les résultats de ces analyses et leurs implications sur la chronologie des réappropriations médiévales de la ville antique.

\section{Contexte archéologiQue}

\section{Four FR59006 de la nécropole de la Porte médiane}

La Porte médiane est une des portes principales des fortifications septentrionales de la ville, de laquelle sortent dès l'époque républicaine trois axes dont la via Domitiana (figure 1). Ce secteur se caractérise à l'époque romaine par la présence de monuments funéraires, dont le mausolée D59 à l'intérieur duquel a été aménagé le four étudié en archéomagnétisme. Les fouilles ont été menées dans un premier temps par l'université de Naples « l'Orientale » en 2006 puis par le Centre Jean Bérard en 2011-2013 (Brun et al., 2013; Brun et al., 2014).

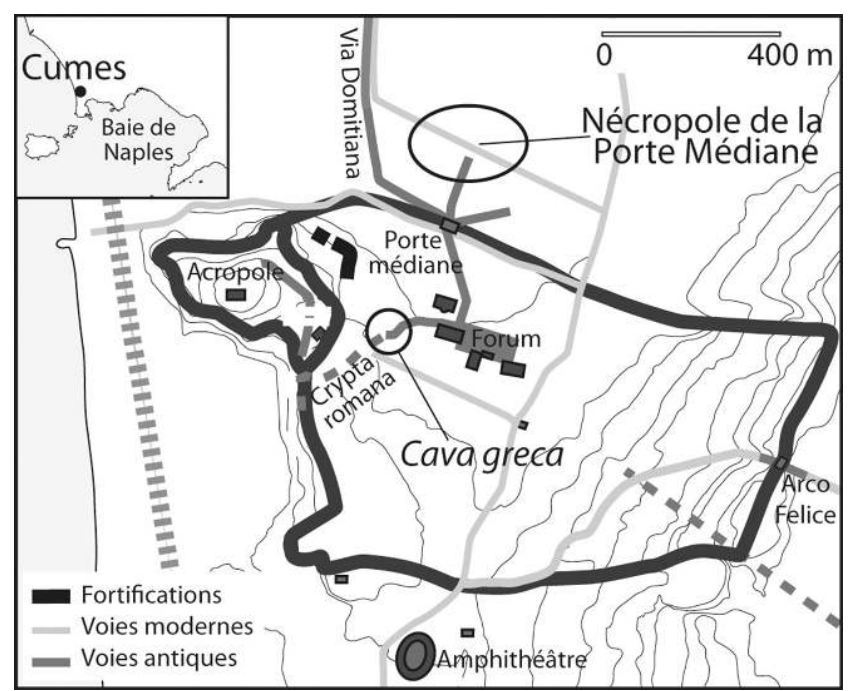

Figure 1 : Plan général de Cumes avec localisation de la Cava greca et de la nécropole de la Porte médiane (ellipses rouges), sites de découverte des deux fours étudiés.

Figure 1: Map of the city of Cumae with localisation of the Cava greca and of the necropolis of the Median Gate (red ellipses), where the two studied kilns have been unearthed.

Le mausolée D59 est situé immédiatement à l'extérieur de la Porte, sur le côté est du grand espace dallé qui est aménagé devant celle-ci à partir de la fin du i ${ }^{\text {er }}$ siècle apr. J.-C. Il s'agit d'un colombarium accueillant dans des niches les crémations de plusieurs individus. La stratigraphie et ses caractéristiques architecturales placent sa construction au II $^{\mathrm{e}}$ siècle apr. J.-C. La fonction funéraire a vraisemblablement été perdue aux $\mathrm{III}^{\mathrm{e}}$ et $\mathrm{IV}^{\mathrm{e}}$ siècles et dans sa deuxième phase d'utilisation le monument funéraire est transformé en espace artisanal. À l'intérieur de la chambre funéraire sont aménagés deux fours, FR59006 et FR59018, installés contre le mur est et sur le sol originel du mausolée (figure 2a). Le four FR59006 est le premier à avoir été construit. Dans un second temps, le four FR59018, plus petit, vient s'appuyer contre la paroi sud du four FR59006. La présence dans le comblement de tegulae et d'imbrices portant des traces de surcuisson ou de malfaçon laisse supposer qu'il s'agit de fours de tuiliers. Les deux fours sont à deux volumes avec une chambre de chauffe rectangulaire. Ils s'apparentent au type IIE de Le $\mathrm{Ny}$ (1988), 4.4.2 de Thuillier (2003) ou encore II/b de Cuomo di Caprio (1971-1972). Dans le cas du four prélevé FR59006, seule la chambre de chauffe a été conservée (figure $2 b$ ). Cette chambre présente quatre murets transversaux, qui supportaient la sole dont seul un lambeau a été préservé. Les parois et les murets des fours sont majoritairement constitués de briques et de tuiles. 

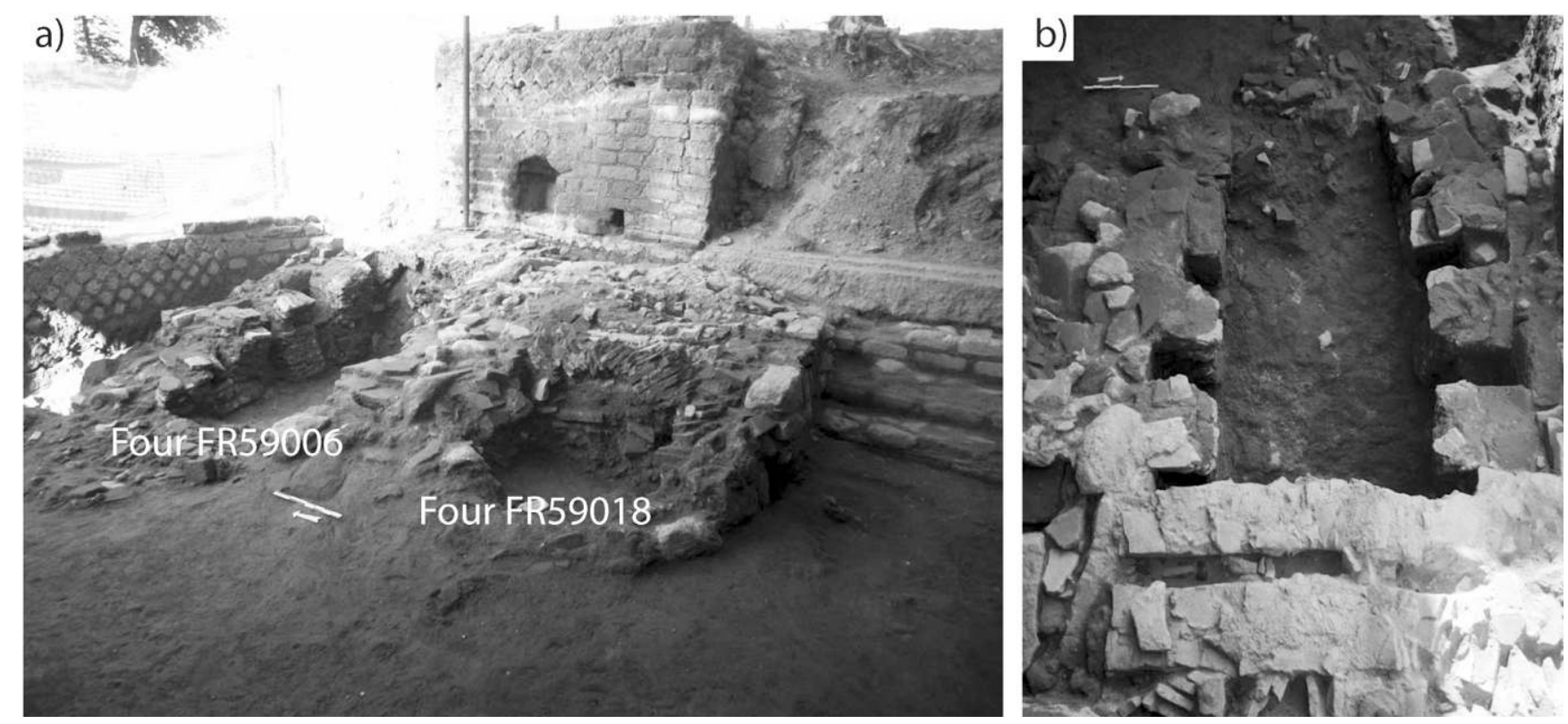

Figure 2 : Le four FR59006 à la fin de la campagne de fouilles 2012. a) Vue générale des deux fours FR59006 et FR59018 aménagés dans le mausolée D59. b) Vue sommitale de la chambre de chauffe du four FR59006. (Clichés Centre Jean Bérard)

Figure 2: The kiln FR59006 at the end of the 2012 excavations. a) General view of the two kilns FR59006 and FR59018 installed inside the mausoleum D59. b) Top view of the heating chamber of the kiln FR59006. (C) Centre Jean-Bérard)

Les deux structures sont scellées par les mêmes couches d'abandon et de destruction. C'est dans ces niveaux qu'ensuite, à une date inconnue mais antérieure au $\mathrm{VII}^{\mathrm{e}}$ siècle apr. J.-C., est implantée une sépulture. Cette tombe est recouverte par plusieurs couches de remblai. La fonction du monument à cette époque reste indéterminée, mais il est possible d'envisager une occupation à caractère également artisanal. L'aspect des soles des fours FR59006 et FR59018 et les niveaux cendreux associés suggèrent une réutilisation des soles comme base de foyer.

Ce phasage date le fonctionnement des fours entre le $v^{e}$ et le $\mathrm{VII}^{\mathrm{e}}$ siècles. Le mobilier archéologique récupéré dans les chambres de chauffe se limite à une monnaie byzantine et à un fragment de sigillée africaine et ne permet pas de préciser cet intervalle chronologique. Dans ce contexte, la datation archéomagnétique du four 59006 constitue un nouvel élément de datation très précieux. Le prélèvement a porté sur les briques et les tuiles à la base des murets de soutènement et des parois de la chambre de chauffe. Le moment daté est la dernière utilisation du four FR59006, qui est probablement contemporaine de celle du four FR59018.

\section{Four de la Cava greca}

La Cava greca (" carrière grecque ») forme le débouché dans la villa basse de la Crypta romana, une galerie souter- raine reliant l'Acropole à la baie située au sud-est du Mont de Cumes (figure 1). Le four a été mis au jour en 2006 sur la bordure orientale de la carrière (Caputo et Regis, 2009). La structure est mieux conservée que les fours du mausolée D59 de la Porte médiane. La chambre de chauffe avec ses trois arcs de soutènement, la sole, la partie basse de la chambre de cuisson et l'alandier ont été préservés (figure 3). Le four a une forme rectangulaire avec une longueur de $2,25 \mathrm{~m}$ et une largeur de 1,80 m. Les parois sont constituées de briques. Comme pour les fours du mausolée D59, leurs dimensions variées indiquent qu'il s'agit de matériaux de récupération. Le prélèvement archéomagnétique s'est focalisé sur les briques de la base de la chambre de cuisson. Plus de 300 tessons de céramique commune ont été retrouvés dans le comblement de la chambre de cuisson. L'absence de traces d'utilisation et l'homogénéité de leur surface suggèrent que cet assemblage de céramique culinaire constitue la production du four.

Le four est associé à un niveau de sol d'époque byzantine, qui est recoupé, à l'autre extrémité de la Cava greca, par une sépulture d'enfant datée des VII et VIII ${ }^{\mathrm{e}}$ siècles apr. J.-C. (Caputo et Regis, 2009). La céramique produite dans le four se rapproche typologiquement de celle découverte sur les sites de Carminiello ai Mannesi (Arthur, 1994), de Piazza Municipio et de Piazza Bovio à Naples (Carsana et al., 2007), ainsi que du mobilier conservé au musée du Sannio à 


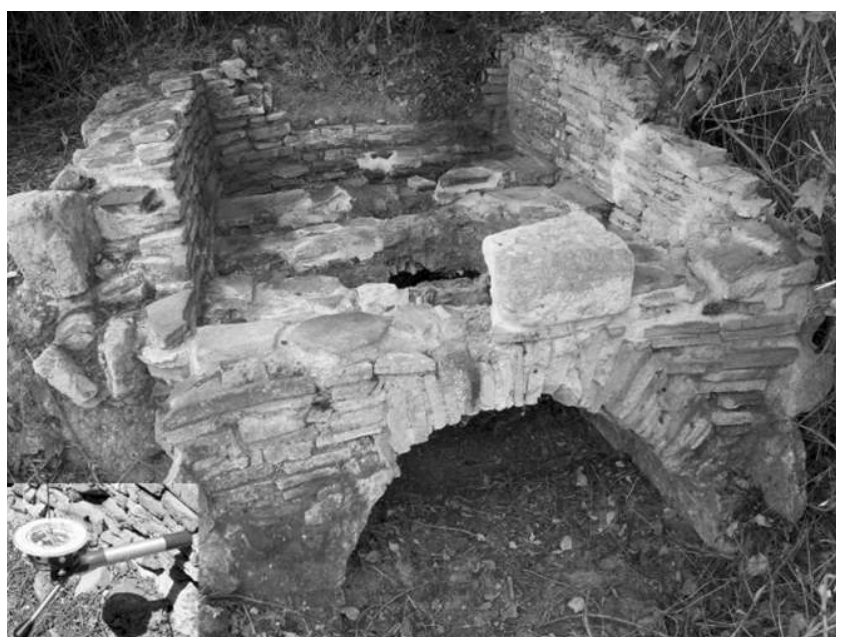

Figure 3 : Four de potier retrouvé près de la Cava greca avec en encart une vue du dispositif d'orientement des prélèvements. (Clichés Gw. Hervé)

Figure 3: Pottery kiln nearby the Cava greca with a detailed view of the orienting tool used during the sampling. (C) Gw. Hervé)

Bénévent. Ces comparaisons régionales placent le fonctionnement du four entre la fin du vi ${ }^{\mathrm{e}}$ et le début du viII ${ }^{\mathrm{e}}$ siècle apr. J.-C.

\section{Méthodes et RÉsultats ARCHÉOMAGNÉTIQUES}

\section{Méthode de prélèvement}

Les briques des parois des deux fours ont été prélevées par carottage ( $25 \mathrm{~mm}$ de diamètre). 26 briques ont été carottées pour le four FR59006 du mausolée D59 et 23 pour le four de la Cava greca. Sur chaque four, 18 carottes ont pu être orientées en place. L'inclinaison et l'orientation au Nord magnétique de chaque carotte ont été reportées avec une tête d'orientement paléomagnétique Pomeroy (figure 3). La présence de structures métalliques au-dessus des deux fours est susceptible de perturber la boussole. Dans le cas du four FR59006, un orientement supplémentaire au Nord géographique via un théodolite-laser a pallié cet inconvénient. Cela n'a pas été envisageable pour le four de la Cava greca du fait de sa situation topographique particulière et une déviation de la déclinaison de ses prélèvements est alors possible. Le prélèvement des deux fours s'est conclu par le rebouchage des briques carottées.

\section{Analyses archéomagnétiques}

Au cours de la dernière utilisation des structures, les briques ont acquis une aimantation thermorémanente (ATR) parallèle et proportionnelle au champ géomagnétique ambiant. L'objectif des analyses archéomagnétiques est de déterminer l'archéodirection (inclinaison et déclinaison) et l'archéointensité de ce champ géomagnétique.

Les analyses ont été menées au laboratoire d'archéomagnétisme de Rennes. Les carottes ont d'abord été coupées en spécimens de $22 \mathrm{~mm}$ de longueur, qui est la taille standard pour les mesures d'aimantation rémanente aux magnétomètres spinner JR6 et cryogénique $2 \mathrm{G}$ que nous avons utilisés.

Les directions d'aimantation rémanente naturelle observées sur chaque four présentent un très bon regroupement, ce qui indique généralement la présence d'une seule composante d'aimantation. Environ un tiers des spécimens (10 pour le four du mausolée D59 et 8 pour celui de la Cava greca) a été soumis à une désaimantation thermique, qui permet de déterminer l'archéodirection et l'autre partie (13 pour le four du mausolée D59 et 14 pour celui de la Cava greca) au protocole de Thellier-Thellier (Thellier et Thellier, 1959), qui fournit aussi l'archéointensité du champ géomagnétique. La désaimantation thermique consiste à chauffer les spécimens par paliers successifs de température (en l'occurrence onze) en champ magnétique nul. Les chauffes ont été réalisées dans un four MMTD60 de Magnetic Measurements. Les résultats obtenus pour l'ensemble des spécimens confirment la présence d'une seule composante d'aimantation rémanente, qui est l'ATR acquise lors de la dernière utilisation des deux fours.

À la différence de la désaimantation thermique, les chauffes du protocole de Thellier-Thellier sont effectuées en présence d'un champ d'intensité connue, ici $60 \mu \mathrm{T}$. Le rapport entre l'ATR de laboratoire ainsi créée et l'ARN ("ATR archéologique »), visualisé sur un diagramme d'Arai, permet de déterminer l'archéointensité (figure 4). Treize paliers de chauffe ont été réalisés entre 100 et $565{ }^{\circ} \mathrm{C}$. Afin de contrôler l'absence de transformations minéralogiques au cours des chauffes successives, des bouclages ont été réalisés tous les deux paliers de température. À cet effet, la susceptibilité en champ faible a aussi été mesurée après chaque étape. Les 27 spécimens analysés sur les deux fours donnent un très bon résultat avec un diagramme ARN-ATR linéaire et une direction d'ARN stable (figure 4). Ils respectent largement les critères d'acceptation généralement préconisés (Genevey et al., 2009; Hervé et al., 2013a; Leonhardt et al., 2004) avec des facteurs de qualité q compris entre 25 et 127 (tableau 1). 
a)

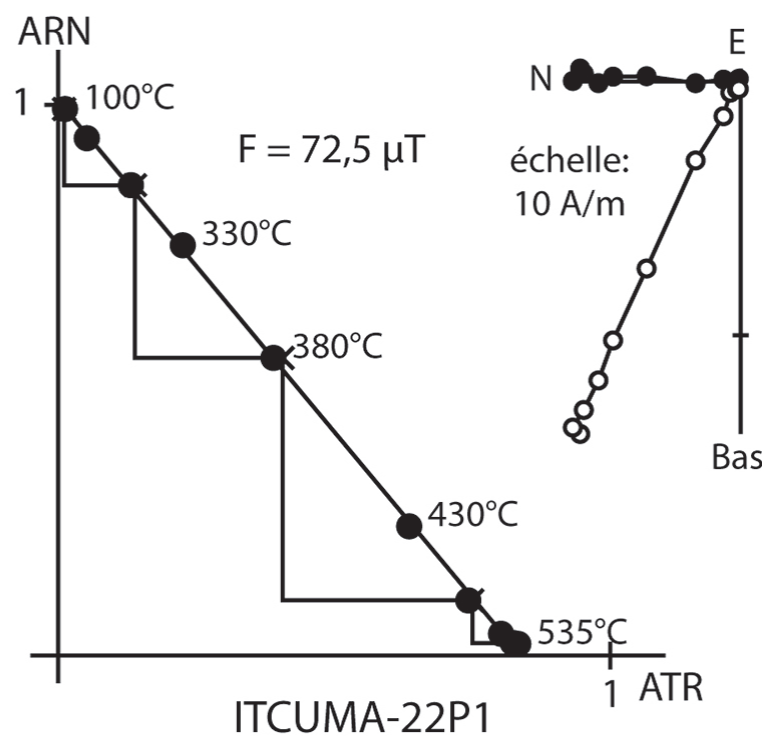

b)

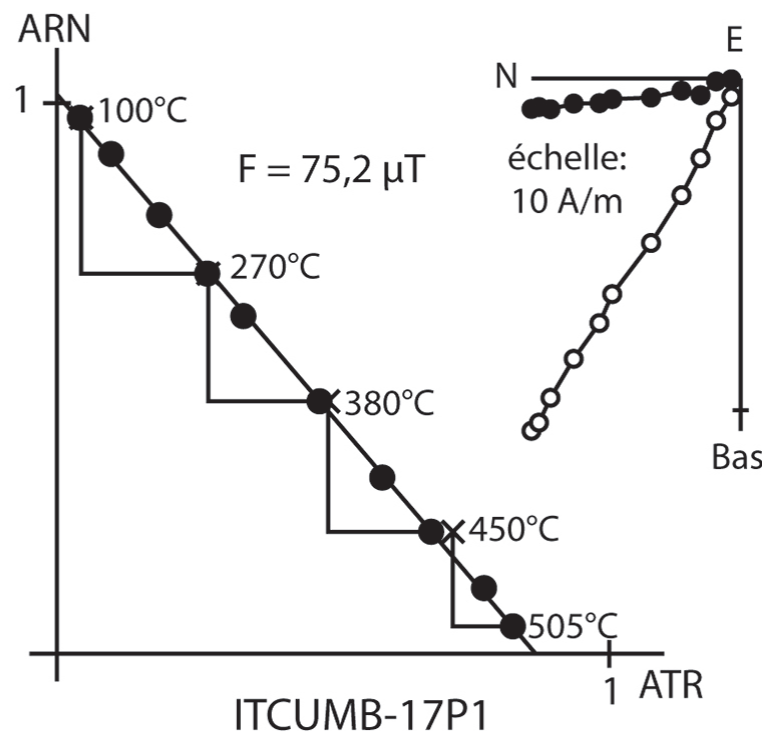

Figure 4 : Exemples de résultats du protocole de Thellier-Thellier pour a) le four FR59006 du mausolée D59 et b) le four de la Cava greca. Sur le diagramme d'Arai à gauche, la pente est proportionnelle à l'archéointensité. La direction en coordonnées in situ est visualisée à droite sur les diagrammes de Zijderveld. Les cercles pleins (vides) sont les projections sur le plan horizontal (vertical). Figure 4: Representative results of Thellier-Thellier protocol for a) the kiln FR59006 from D59 mausoleum and b) the kiln of the Cava greca. On the Arai diagrams left, the slope is proportional to the archaeointensity. The direction in in situ coordinates is shown on the right in the Zijderveld diagrams. Solid (open) circles are projections upon horizontal (vertical) planes.
En raison de directions d'aimantation préférentielles dans les minéraux ferromagnétiques, l'ATR peut être déviée et son intensité modifiée par rapport au champ magnétique ambiant lors de la dernière utilisation. L'effet d'anisotropie, qui est notamment significatif pour les briques et autres terres cuites architecturales, est lié aux gestes des artisans potiers lors du façonnage (Kovacheva et al., 2009; Veitch et al., 1984). Cet effet est corrigé en déterminant pour chaque spécimen son tenseur d'anisotropie d'ATR. Ce protocole consiste en six chauffes successives selon les axes $+Z,-Z,+X$, $-X,+Y$ et $-Y$ des spécimens, suivi d'un bouclage selon $+Z$ pour vérifier l'absence de transformations minéralogiques (Chauvin et al., 2000). Ce protocole a été effectué à 480 ou $510{ }^{\circ} \mathrm{C}$ sur les spécimens soumis à la désaimantation thermique ou au protocole de Thellier-Thellier. Pour les briques des deux fours de Cumes, les pourcentages d'anisotropie sont compris entre 5 et $40 \%$. L'archéodirection et l'archéointensité de chaque spécimen ont été individuellement corrigées.

L'intensité de l'ATR dépend aussi de la vitesse de refroidissement (Néel, 1955; Fox et Aitken, 1980). Or, au cours du protocole de Thellier-Thellier, l'ATR est acquise sur environ une demi-heure, soit sur une durée bien plus courte que le refroidissement archéologique initial. Les archéointensités sont alors biaisées (Chauvin et al., 2000; Genevey et Gallet, 2002). Elles ont été corrigées en suivant le protocole de Gómez-Paccard et al. (2006) avec un refroidissement lent d'environ 25 heures $\left(0,3{ }^{\circ} \mathrm{C} / \mathrm{min}\right)$. Le refroidissement lent est suivi d'un dernier cycle de chauffe avec refroidissement rapide pour vérifier l'absence d'évolutions minéralogiques. La correction n'est pas appliquée lorsque le facteur d'altération est supérieur en valeur absolue au facteur de correction de la vitesse de refroidissement. Les facteurs de correction des autres spécimens des deux fours sont compris entre -5 et $6 \%$. Les archéointensités corrigées des effets de l'anisotropie d'ATR et de la vitesse de refroidissement sont présentées dans le tableau 1.

\section{Archéodirections et archéointensités moyennes}

La moyenne d'archéointensité des deux fours a été pondérée par le facteur de qualité q (Prévot, 1985). Larchéointensité moyenne du four FR59006 du mausolée D59 est égale à $70,1 \pm 4,4 \mu \mathrm{T}$ et celle du four de la Cava greca à $74,1 \pm 4,2 \mu \mathrm{T}$. Pour les deux fours, l'application des corrections d'anisotropie et de vitesse de refroidissement a réduit la déviation standard d'environ $30 \%$.

Les diagrammes avec projection stéréographique de la figure 5 montrent la distribution par four des archéodirections des 18 spécimens orientés in situ. Le caractère 


\begin{tabular}{|c|c|c|c|c|c|c|c|c|c|c|c|}
\hline \multicolumn{4}{|c|}{ Four FR59006 du mausolée D59 } & \multirow{2}{*}{$\operatorname{Mad}\left({ }^{\circ}\right)$} & \multirow{2}{*}{ Dang $\left(^{\circ}\right)$} & \multirow{2}{*}{$\mathrm{f}$} & \multirow{2}{*}{$\mathrm{g}$} & \multirow{2}{*}{$\mathrm{q}$} & \multirow{2}{*}{$ß$} & \multirow{2}{*}{$\mathrm{F}_{\mathrm{a}}(\mu \mathrm{T})$} & \multirow{2}{*}{$\mathrm{F}_{\mathrm{atc}}(\mu \mathrm{T})$} \\
\hline Nom & Et. 1 - Et. $2\left({ }^{\circ} \mathrm{C}\right)$ & $\mathrm{n}$ & $\mathrm{F}(\mu \mathrm{T})$ & & & & & & & & \\
\hline ITCUMA-2P1 & $100-535$ & 10 & 63,6 & 1,1 & 0,3 & 0,84 & 0,87 & 51,0 & 0,014 & 63,2 & 60,3 \\
\hline ITCUMA-4P1 & $100-535$ & 10 & 68,6 & 1,8 & 0,7 & 0,91 & 0,84 & 54,4 & 0,014 & 70,7 & 68,6 \\
\hline ITCUMA-5P1 & $100-565$ & 13 & 74,6 & 0,8 & 0,8 & 0,83 & 0,90 & 80,3 & 0,009 & 69,8 & 67,5 \\
\hline ITCUMA-6P1 & $100-545$ & 12 & 61,4 & 1,2 & 0,5 & 0,83 & 0,89 & 77,2 & 0,010 & 64,0 & 67,4 \\
\hline ITCUMA-7P1 & $160-545$ & 11 & 69,7 & 1,1 & 0,5 & 0,89 & 0,89 & 94,3 & 0,008 & 70,5 & 70,2 \\
\hline ITCUMA-9P1 & $200-535$ & 9 & 72,2 & 1,6 & 0,9 & 0,75 & 0,76 & 31,5 & 0,018 & 77,0 & 75,0 \\
\hline ITCUMA-12P1 & $100-535$ & 10 & 75,6 & 0,9 & 0,1 & 0,98 & 0,68 & 59,7 & 0,011 & 75,4 & 71,7 \\
\hline ITCUMA-13BP1 & $100-505$ & 10 & 79,4 & 2,1 & 2,4 & 0,87 & 0,85 & 29,6 & 0,025 & 74,5 & 74,1 \\
\hline ITCUMA-17P1 & $100-535$ & 10 & 59,6 & 1,4 & 1,0 & 0,94 & 0,86 & 46,2 & 0,018 & 74,1 & 64,2 \\
\hline ITCUMA-20P1 & $100-535$ & 10 & 69,4 & 2,1 & 1,1 & 0,92 & 0,86 & 39,6 & 0,020 & 63,3 & 68,6 \\
\hline ITCUMA-22P1 & $100-535$ & 10 & 72,2 & 1,0 & 0,4 & 0,97 & 0,81 & 103,8 & 0,008 & 72,8 & 72,5 \\
\hline ITCUMA-24P1 & $200-535$ & 9 & 81,3 & 1,0 & 1,3 & 0,80 & 0,85 & 95,8 & 0,011 & 80,4 & 74,1 \\
\hline ITCUMA-25P1 & $160-505$ & 9 & 75,7 & 2,1 & 0,7 & 0,94 & 0,78 & 67,0 & 0,011 & 74,9 & 74,9 \\
\hline \multicolumn{12}{|c|}{$\mathrm{F}_{\text {moy }}=70,1 \mu \mathrm{T} \mathrm{SD}=4,4 \mu \mathrm{T} \mathrm{N}=13$ spécimens } \\
\hline \multicolumn{2}{|c|}{ Four de la Cava greca } & \multirow{2}{*}{$\mathrm{n}$} & \multirow{2}{*}{$\mathrm{F}(\mu \mathrm{T})$} & \multirow{2}{*}{$\operatorname{Mad}\left({ }^{\circ}\right)$} & \multirow{2}{*}{ Dang $\left(^{\circ}\right)$} & \multirow{2}{*}{$\mathrm{f}$} & \multirow{2}{*}{$\mathrm{g}$} & \multirow{2}{*}{$\mathrm{q}$} & \multirow{2}{*}{$ß$} & \multirow{2}{*}{$\mathrm{F}_{\mathrm{a}}(\mu \mathrm{T})$} & \multirow{2}{*}{$\mathrm{F}_{\mathrm{atc}}(\mu \mathrm{T})$} \\
\hline Nom & Et. 1 - Et.2 $\left({ }^{\circ} \mathrm{C}\right)$ & & & & & & & & & & \\
\hline ITCUMB-1P1 & $100-505$ & 10 & 74,2 & 1,1 & 0,8 & 0,82 & 0,88 & 75,4 & 0,009 & 75,5 & 75,5 \\
\hline ITCUMB-4P1 & $100-505$ & 10 & 82,9 & 1,0 & 0,7 & 0,83 & 0,86 & 63,7 & 0,011 & 79,0 & 74,3 \\
\hline ITCUMB-5P1 & $100-525$ & 11 & 68,9 & 1,2 & 1,1 & 0,88 & 0,89 & 59,7 & 0,013 & 73,5 & 73,5 \\
\hline ITCUMB-6P1 & $100-505$ & 10 & 70,9 & 1,1 & 1,1 & 0,88 & 0,88 & 69,4 & 0,011 & 74,8 & 72,9 \\
\hline ITCUMB-7P1 & $100-505$ & 10 & 78,5 & 1,2 & 2,0 & 0,83 & 0,87 & 25,5 & 0,028 & 81,9 & 76,6 \\
\hline ITCUMB-8P1 & $100-505$ & 10 & 75,3 & 1,1 & 1,0 & 0,89 & 0,86 & 50,5 & 0,015 & 78,9 & 78,9 \\
\hline ITCUMB-9P1 & $100-505$ & 10 & 65,1 & 1,2 & 1,2 & 0,86 & 0,87 & 46,5 & 0,016 & 66,2 & 65,8 \\
\hline ITCUMB-11P1 & $100-505$ & 10 & 75,6 & 1,1 & 0,6 & 0,95 & 0,84 & 70,7 & 0,011 & 78,5 & 75,6 \\
\hline ITCUMB-12P1 & $100-505$ & 10 & 64,8 & 1,3 & 0,7 & 0,95 & 0,87 & 28,1 & 0,029 & 66,2 & 65,1 \\
\hline ITCUMB-15P1 & $100-505$ & 10 & 76,7 & 0,9 & 0,9 & 0,83 & 0,88 & 50,6 & 0,014 & 78,2 & 77,3 \\
\hline ITCUMB-17P1 & $100-505$ & 10 & 70,3 & 1,2 & 1,1 & 0,90 & 0,88 & 126,8 & 0,006 & 74,5 & 75,2 \\
\hline ITCUMB-21P1 & $100-505$ & 10 & 82,1 & 1,1 & 0,8 & 0,80 & 0,88 & 61,8 & 0,011 & 74,7 & 75,1 \\
\hline ITCUMB-22P1 & $100-505$ & 10 & 77,1 & 1,4 & 0,4 & 0,86 & 0,86 & 42,9 & 0,017 & 74,2 & 78,1 \\
\hline ITCUMB-23P1 & $100-505$ & 10 & 72,0 & 1,0 & 0,8 & 0,89 & 0,87 & 49,1 & 0,016 & 70,1 & 73,3 \\
\hline \multicolumn{12}{|c|}{$\mathrm{F}_{\text {moy }}=74,1 \mu \mathrm{T} \mathrm{SD}=4,2 \mu \mathrm{T} \mathrm{N}=14$ spécimens } \\
\hline
\end{tabular}

Tableau 1 : Résultats d'archéointensité. Dans l'ordre des colonnes : le nom du spécimen; l'intervalle de température sur lequel est calculée l'archéointensité; le nombre n de paliers de chauffe; l'archéointensité F sans corrections d'anisotropie et de vitesse de refroidissement; les paramètres $\mathrm{MAD}$ " maximum angular deviation » et DANG « deviation angle " quantifiant la linéarité de la direction et son caractère primaire; la fraction d'ARN f; le facteur " gap " g; le facteur de qualité de l'archéointensité q (Coe et al., 1978); le rapport ß de l'erreur standard de la pente sur la valeur absolue de la pente du diagramme d'Arai; l'archéointensité $\mathrm{F}_{\mathrm{a}}$ corrigée de l'anisotropie d'aimantation thermorémanente; l'archéointensité $\mathrm{F}_{\text {a+c }}$ corrigée de l'anisotropie et de la vitesse de refroidissement. En-dessous, est indiquée l'archéointensité moyenne par structure avec la déviation standard et le nombre de spécimens.

Table 1: Archaeointensity results. Columns from left to right: specimen name; temperature interval used to determine intensity; number $n$ of temperature steps within this temperature range; archaeointensity F without any corrections; Maximum Angular Deviation; Deviation Angle; NRM fraction f; gap factor g; quality factor $q$ (Coe et al., 1978); Ratio $\beta$ of the standard error of the slope to the absolute value of the slope;

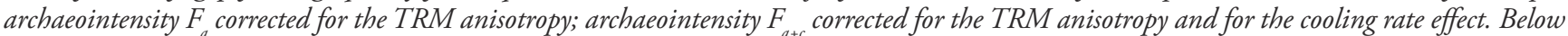
is indicated the mean archaeointensity with its standard deviation and the number of specimens. 
fishérien des distributions a été vérifié par le test de Fisher et al. (1987). Les paramètres de confiance de la direction d'aimantation moyenne sont calculés par la statistique de Fisher (1953) (figure 5). Pour les deux structures, la correction d'anisotropie améliore le regroupement des spécimens comme le révèle l'augmentation du facteur $\mathrm{k}$. Après correction, la valeur d'environ 360 indique un très bon regroupement. Dans le cas du four de la Cava greca, la déclinaison moyenne est égale à $-15,5^{\circ}$. En Europe, de telles valeurs occidentales ne sont observées qu'entre le Xvir ${ }^{\mathrm{e}}$ et le $\mathrm{XIX}^{\mathrm{e}}$ siècle apr. J.-C. (Hervé et al., 2013b) en totale contradiction avec le contexte archéologique et historique de ce four. Cette erreur systématique s'explique vraisemblablement par une déviation locale de la boussole lors de l'orientement à cause de la structure métallique de protection du four. De ce fait, la datation archéomagnétique de ce four ne pourra reposer que sur l'inclinaison et l'archéointensité. Les carottes du four FR59006 ayant pu être orientées également au théodolite laser, la déclinaison de leur ATR ne peut être affectée par un tel phénomène et la datation sera effectuée avec les trois paramètres du champ géomagnétique.

\section{Datation archéomagnétique}

La datation archéomagnétique repose sur la comparaison de l'archéodirection et de l'archéointensité avec leur référentiel. Les référentiels les plus usités sont les courbes de variation séculaire régionales (par exemple, Gallet et al., 2002, Genevey et al., 2013, Hervé et al., 2013a et b en France; Schnepp et Lanos, 2005 en Allemagne; Kovacheva et al., 2014 en Bulgarie). Ces courbes sont définies en un lieu de référence et sont construites à partir de données de référence situées au maximum à $1000 \mathrm{~km}$ du lieu choisi.

En Italie, malgré un potentiel indéniable, le nombre de données de référence est faible en comparaison des autres pays européens. Les actuelles courbes de variation séculaire de la direction et de l'intensité en Italie demeurent incomplètes (Tema et al., 2013) ou présentent une large enveloppe d'erreur qui les rend peu informatives à des fins de datation archéomagnétique (Tema et al., 2006). L'alternative est l'utilisation du modèle géomagnétique européen SCHA.DIF.3k (Pavón-Carrasco et al., 2009; Pavón-Carrasco et al., 2014). Ce modèle, fondé sur une description en harmoniques sphériques régionales ("Spherical Cap Harmonic Analysis »), rend compte à la fois de la variation spatiale et de la variation temporelle du champ géomagnétique à l'échelle de l'Europe entière. Il est construit par inversion à partir des données de référence disponibles. La datation archéomagnétique avec la déclinaison et l'inclinaison est réalisée avec les prédictions à
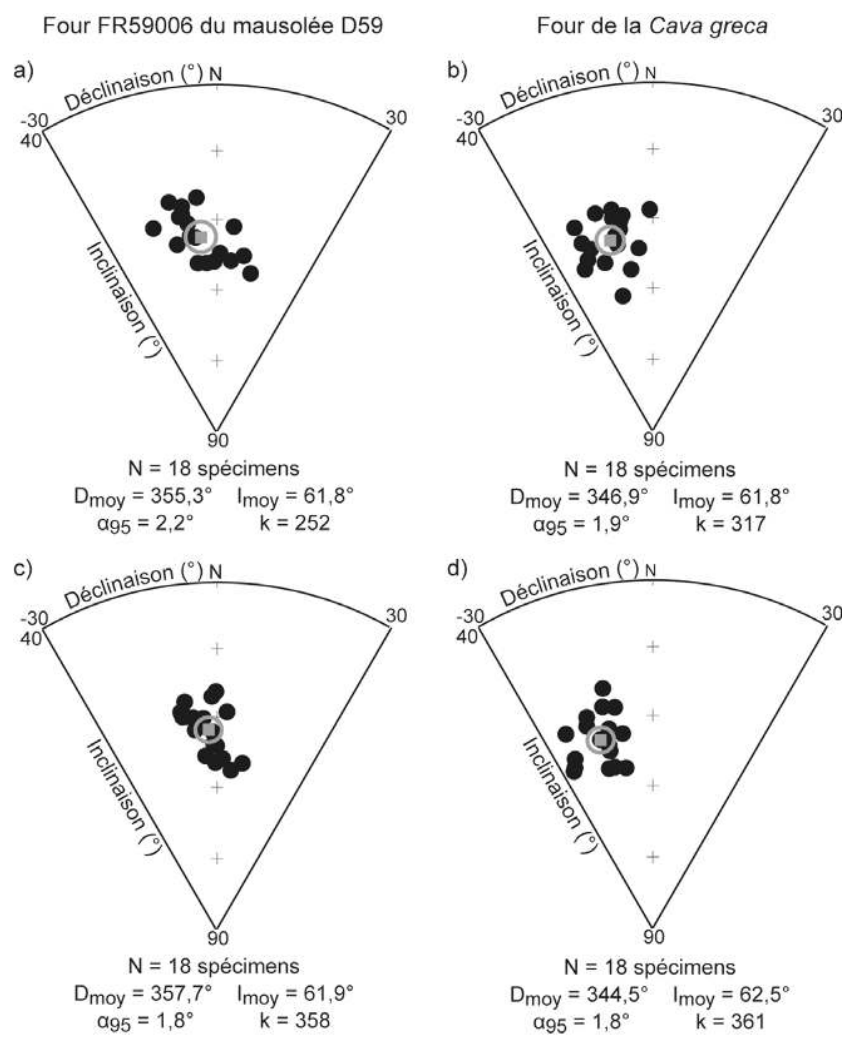

Figure 5 : Stéréogrammes des archéodirections sans (a-b) et avec (c-d) correction de l'anisotropie d'ATR. La direction moyenne est indiquée en gris avec son erreur à $95 \%$ de confiance $\left(\alpha_{95}\right)$.

Figure 5: Stereographic plots of the archaeodirections without ( $a-b)$ and with $(c-d)$ correction of the TRM anisotropy. The mean direction is plotted in grey with its error at 95 per cent of confidence $\left(\alpha_{95}\right)$.

Cumes du modèle originel SCHA.DIF.3k (Pavón-Carrasco et al., 2009). Pour l'intensité, le modèle a été réactualisé avec une sélection des archéointensités de référence en fonction de la qualité de leur protocole d'acquisition (Pavón-Carrasco et al., 2014). Nous avons choisi la prédiction à Cumes de leur modèle $\mathrm{C}$, car il offre un bon compromis entre la qualité et le nombre des données de référence : le modèle est construit sur un nombre élevé de données tout en éliminant celles, en général les plus anciennement acquises, de moins bonne qualité.

En pratique, la datation archéomagnétique consiste à reporter l'inclinaison, la déclinaison (seulement pour le four FR59006 du mausolée D59) et l'archéointensité sur leur courbe de référence respective (figures 6 et 7). Une densité de probabilité de date est obtenue pour chaque paramètre. Comme la direction et l'intensité ont pu reprendre plusieurs fois la même valeur au cours du temps, ces densités sont généralement multimodales. Le résultat final de la datation est un (ou des) intervalle(s) de date à $95 \%$ de confiance 

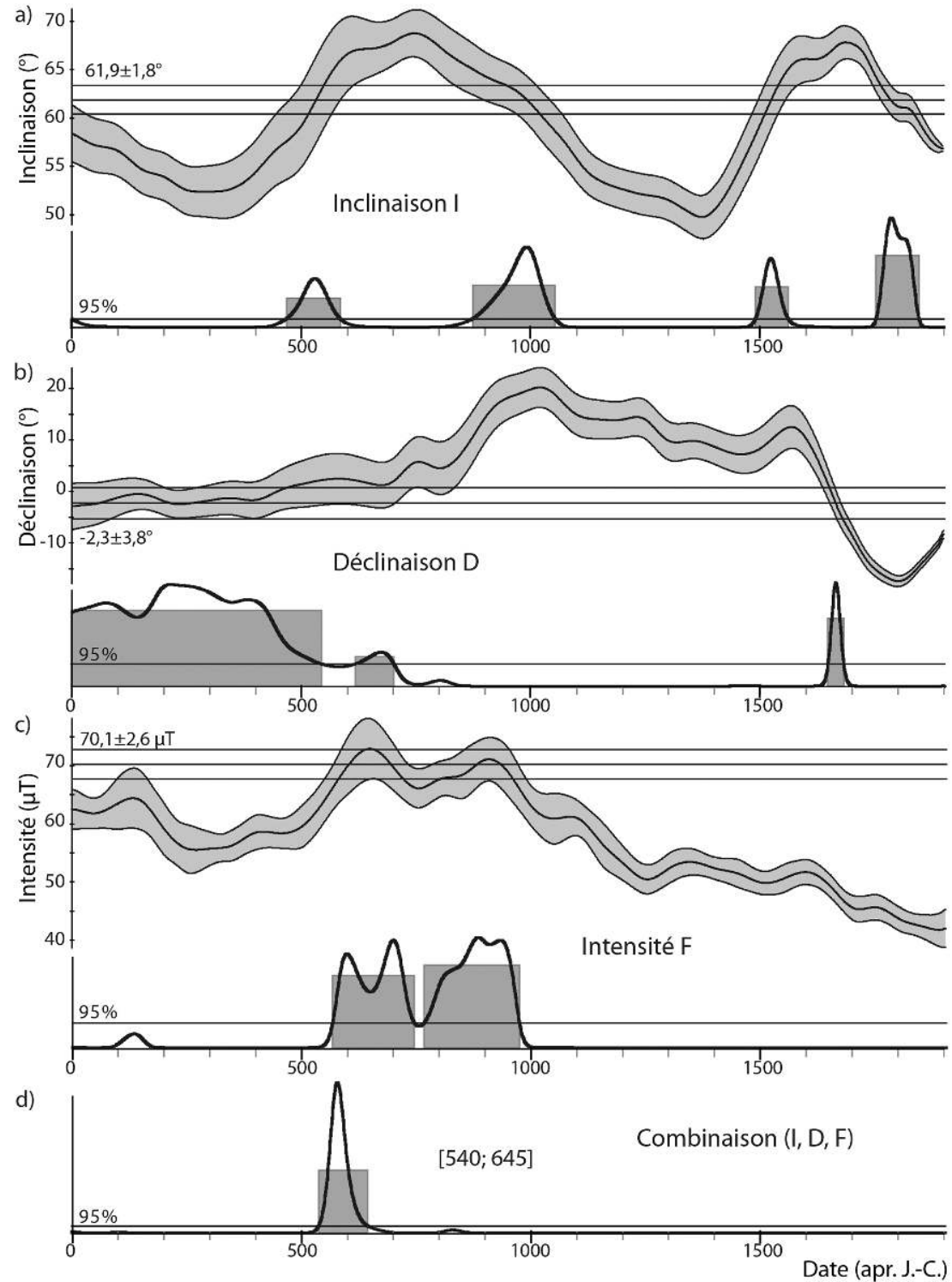

Figure 6 : Datation archéomagnétique du four FR59006 du mausolée D59. L'inclinaison (a), la déclinaison (b) et l'archéointensité (c) moyennes avec leur erreur à $95 \%$ de confiance sont comparées à la prédiction à Cumes du modèle géomagnétique européen SCHA. DIF.3k. Les trois densités de probabilité de date sont ensuite combinées (d). L'intervalle de date à $95 \%$ de confiance, $[540 ; 645]$ apr. J.-C., est défini à partir de cette combinaison. Figure 6: Archaeomagnetic dating of the kiln FR59006 from the D59 mausoleum. Mean inclination (a), declination (b) and archaeointensity (c) are compared to the prediction at Cuma of the European geomagnetic model SCHA.DIF.3k. The three obtained probability densities of date are next combined $(d)$. The interval of date at 95 per cent of confidence, [540;645] AD, is calculated from this combination. calculé sur la combinaison (c'est-à-dire le produit) des deux ou trois densités.

Dans le cas du four FR59006 du mausolée D59, on observe une bonne concordance entre les densités de probabilité de la déclinaison, de l'inclinaison et de l'intensité (figure 6). Leur combinaison donne un seul intervalle de date à $95 \%$ de confiance, $[540 ; 645]$ apr. J.-C. À $68 \%$ de confiance, l'intervalle est $[560 ; 600]$ apr. J.-C. Les densités de probabilité de l'inclinaison et de l'intensité du four de la Cava greca concordent également. La forme bimodale de la combinaison résulte en deux intervalles de date à $95 \%$ de confiance $[590 ; 640]$ et $[860 ; 960]$ apr. J.-C. (figure 7).

\section{Discussion ET CONCLUSION}

En premier lieu, ces résultats illustrent le potentiel de datation de l'intensité au haut Moyen-Age grâce à sa forte variation séculaire (Gómez-Paccard et al., 2012). L'intensité est évidemment très utile, lorsque l'inclinaison ou la déclinaison n'est pas utilisable (figure 7). Dans le cas du four FR59006, l'ajout de l'intensité précise la datation, [435; 600] apr. J.-C. à $95 \%$ de confiance, obtenue avec la direction seule (combinaison de l'inclinaison et de la déclinaison). Cependant, la datation avec l'intensité seule demeure encore peu informative car l'erreur sur ses référentiels reste 
Figure 7 : Datation archéomagnétique du four de la Cava greca. L'inclinaison (a) et l'archéointensité (b) moyennes sont comparées à leur prédiction à Cumes du modèle SCHA. DIF.3k. Les deux densités de probabilité de date sont ensuite combinées (c). Deux intervalles de date à $95 \%$ de confiance, [590; 640] et [860; 960] apr. J.-C., sont obtenus. Figure 7: Archaeomagnetic dating of the kiln from the Cava greca. Mean inclination (a) and archaeointensity (b) are compared to their prediction at Cuma by SCHA.DIF.3k model. The two probability densities of date are combined. Two intervals of date at $95 \%$ of confidence are obtained: [590; 640] and [860; 960] AD.
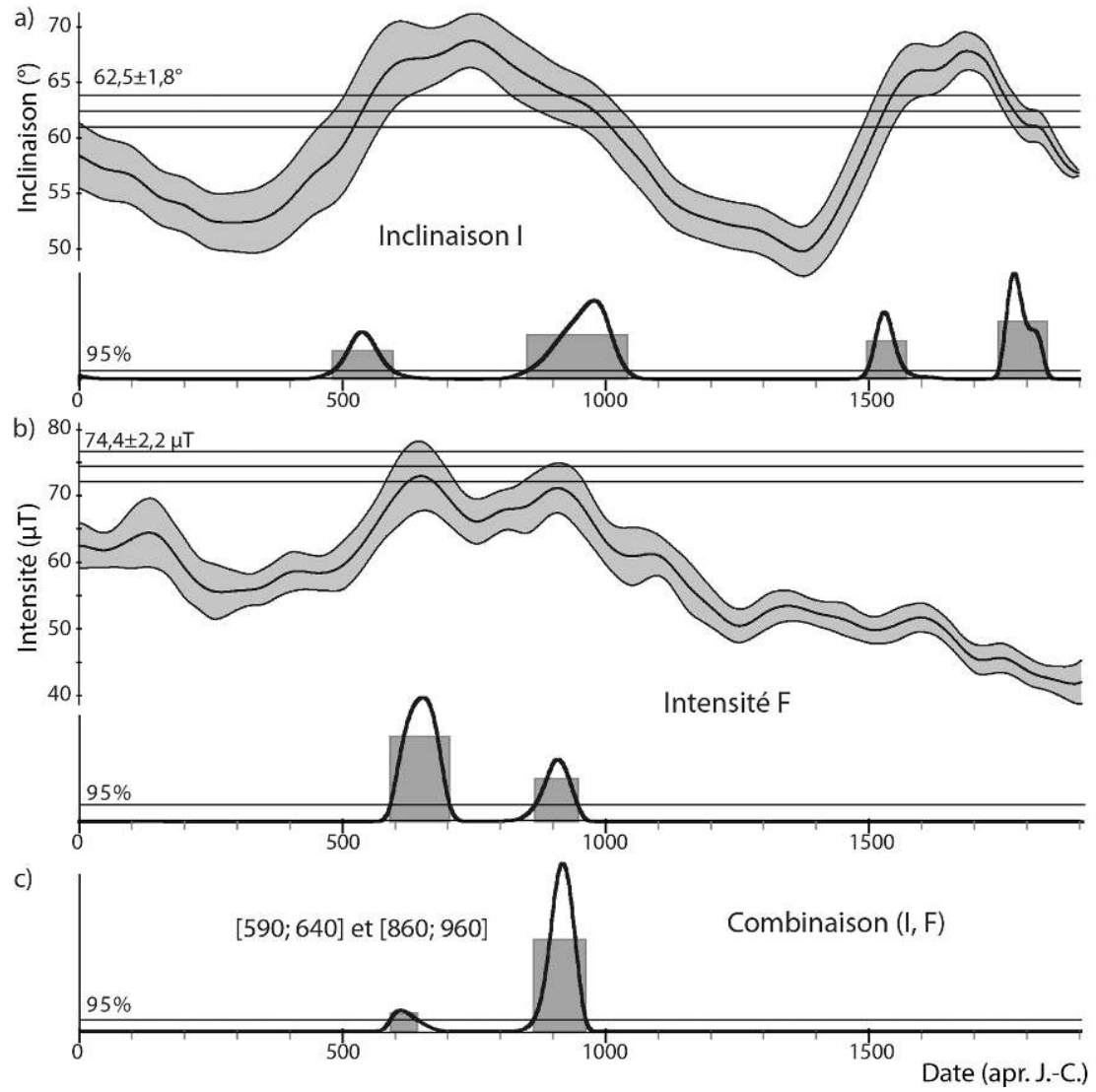

importante (figures 6 et 7). Seule l'acquisition de nouvelles archéointensités de référence au haut Moyen Âge en Europe permettra à l'avenir d'obtenir des intervalles de date plus précis.

Les datations archéomagnétiques confortent et précisent les informations chronologiques apportées par la stratigraphie et la typochronologie. Dans le cas du four de la Cava greca, l'intervalle de date archéomagnétique le plus récent, [860; 960], est incompatible avec la stratigraphie, le sol associé au four étant recoupé par une sépulture datée des $\mathrm{VII}^{\mathrm{e}}$ et $\mathrm{VIII}^{\mathrm{e}}$ siècles. Cet intervalle est aussi en contradiction avec la typochronologie régionale (Caputo et Regis, 2009). Seul l'intervalle de date le plus ancien apparaît possible. Le four de la Cava greca a ainsi cessé de fonctionner entre 590 et 640 apr. J.-C. à $95 \%$ de confiance. L'archéomagnétisme réduit clairement l'intervalle de la datation typochronologique entre la fin du vi ${ }^{\mathrm{e}}$ siècle et le début du viII ${ }^{\mathrm{e}}$ siècle.

Pour le secteur du mausolée D59 à la Porte médiane, l'archéomagnétisme date l'abandon du four FR59006, et probablement aussi celui du four contigu FR59018, entre le milieu du $\mathrm{VI}^{\mathrm{e}}$ et le milieu du viI ${ }^{\mathrm{e}}$ siècle apr. J.-C. La datation archéomagnétique précise considérablement la datation stratigraphique entre le $\mathrm{v}^{\mathrm{e}}$ et le $\mathrm{vII}^{\mathrm{e}}$ siècles. Si les intervalles de date à $95 \%$ de confiance des deux fours étudiés se chevauchent, un test de Student montre que les archéointensités moyennes diffèrent à $95 \%$ de confiance. L'arrêt du fonctionnement du four du mausolée D59 ne peut être contemporain de celui du four de la Cava greca. L'archéointensité plus faible obtenue sur le four FR59006 du mausolée D59 indique qu'il est plus ancien. Aussi, la seconde moitié du $\mathrm{VI}^{\mathrm{e}}$ siècle, qui correspond d'ailleurs à son intervalle de date à $68 \%$ de confiance, apparaît sa datation la plus probable.

À l'échelle de la ville, les datations archéomagnétiques placent le fonctionnement des fours à la période byzantine. Le développement d'activités artisanales durant cette période est révélateur d'une nouvelle prospérité de Cumes, après les conflits entre l'Empire byzantin et les Goths. 


\section{Remerciements}

Les recherches à l'extérieur de la Porte Médiane de Cumes ont été poursuivies grâce aux crédits du ministère des Affaires Etrangères et en collaboration avec la Soprintendenza Speciale per $i$ Beni archeologici di Napoli e Pompei et l'Ufficio per $i$ Beni archeologici di Cuma. Nous remercions Guilhem Chapelin (Centre Jean Bérard) pour son aide précieuse lors du prélèvement archéomagnétique et Philippe Cullerier (Laboratoire de Paléo-Archéomagnétisme, Rennes) pour avoir participé à l'acquisition des archéointensités. Notre gratitude s'adresse également à Francesco-Javier Pavon-Carrasco pour le calcul à Cumes du modèle SCHA.DIF.3k. Enfin, nous remercions les deux relecteurs pour les améliorations apportées à cet article et Julie Simon pour le travail d'édition.

\section{Bibliographie}

Arthur P. (ed.), 1994, Il Complesso Archeologico di Carminiello ai Mannesi, Napoli. Scavi 1983-1984, Galatina.

Brun J.-P., Munzi Pr., Cavassa L., Chapelin G., Cormier A., Duday H., Gualandi S., Le Berre S., Lemaire B., Meluzis N., Neyme D., Piffeteau J.-M., Watel A., 2013. Cumes, Chronique des activités archéologiques de l'École française de Rome, mis en ligne le 28 juin 2013. URL : [http://cefr.revues. org/989].

Brun J.-P., Munzi Pr., Cavassa L., Chapelin G., Duneufjardin P., Le Berre S., Mailleur S., Meluzirs N., Neyme D., Piffeteau J.-M., Watel A., 2014. Cumes, Chronique des activités archéologiques de l'École française de Rome, mis en ligne le 05 février 2014. URL: [http://cefr.revues.org/1076X].

Caputo P., Regis C., 2009. Rinvenimento di una fornace d'età bizantina a Cuma. In PASQUALINI M. (éd.), Les céramiques communes d'Italie et de Narbonnaise : structures de production, typologies et contextes inédits : It s. av. J.-C.-III s. apr. J.-C., Centre Jean-Bérard, Naples, p. 109-115.

Carsana V., D’Amico V., del Vecchio F., 2007. Nuovi dati ceramologici per la storia economica di Napolitra tarda antichatà ed altomidioevo. In M. Bonifay, J.C. Tréglia, Late Roman Coarse Wares, Cooking Wares and Amphorae in the Mediterranean, BAR International Series 1662, 423-438.

Chauvin A., Garcia Y., Lanos P., Laubenheimer F., 2000. Paleointensity of the geomagnetic field recovered on archaeomagnetic sites from France. Physics of the Earth and Planetary Interiors, 120, p. 111-136.

Coe R. S., Grommé S., Mankinen E. A., 1978. Geomagnetic palaeointensities from radiocarbon-dated lava flows on Hawaii and the question of the Pacific non-dipole low. Journal of Geophysical Research, 83, p. 1740-1756.
Cuomo di Caprio N., 1971-1972. Proposta di classificazione delle fornaci per ceramica e laterizi nell'area italiana dalla preistoria a tutta l'epoca romana. Sibrium, 11, p. 371-414.

Fisher R. A., 1953. Dispersion on a sphere. Proceedings of the Royal Society of London, A-127, p. 295-305.

Fisher N.I., Lewis T., Embleton B.J.J., 1987. Statistical Analysis of Spherical Data, Cambridge University Press, 329 p.

Fox J. M. W., Aitken M. J., 1980. Cooling rate dependence of the thermoremanent magnetization. Nature, 283, p. 462-463.

Gallet Y., Genevey A., Le Goff M., 2002. Three millennia of directional variation of the Earth's magnetic field in western Europe as revealed by archeological artefacts. Physics of the Earth and Planetary Interiors, 131 (1), p. 81-89.

Genevey A., Gallet Y., 2002. Intensity of the geomagnetic field in western Europe over the past 2000 years: New data from ancient French pottery. Journal of Geophysical Research, 107 (B11), 2285. doi:10.1029/2001JB000701.

Genevey A., Gallet Y., Rosen J., Le Goff M., 2009. Evidence for rapid geomagnetic field intensity variations in Western Europe over the past 800 years from new French archeointensity data. Earth and Planetary Science Letters, 284 (1-2), p. 132-143.

Genevey A., Gallet Y., Thébault E., Jesset S., Le Goff M., 2013. Geomagnetic field intensity variations in Western Europe over the past 1100 years. Geochemistry, Geophysics, Geosystems, 14 (8), p. 2858-2872.

Gómez-Paccard M., Chauvin A., Lanos P., Dufresne P., Kovacheva M., Hill M. J., Beamud E., Blain S., Bouvier A., Guibert P., 2012. Improving our knowledge of rapid geomagnetic field intensity changes observed in Europe between 200 and 1400 AD. Earth and Planetary Science Letters, 355356, p. 131-143.

Gómez-Paccard M., Chauvin A., Lanos P., Thiriot J., JiménezCastillo P., 2006. Archeomagnetic study of seven contemporaneous kilns from Murcia (Spain). Physics of the Earth and Planetary Interiors, 157 (1-2), p. 16-32.

Hervé G., Chauvin A., Lanos P., 2013a. Geomagnetic field variations in Western Europe from 1500BC to 200AD. Part II: New intensity secular variation curve. Physics of the Earth and Planetary Interiors, 218, p. 51-65.

Hervé G., Chauvin A., Lanos P., 2013b. Geomagnetic field variations in Western Europe from 1500BC to 200AD. Part I: Directional secular variation curve. Physics of the Earth and Planetary Interiors, 218, p. 1-13.

Kovacheva M., Chauvin A., Jordanova N., Lanos P., Karloukovski V., 2009. Remanence anisotropy effect on the palaeointensity results obtained from various archaeological materials, excluding pottery. Earth Planets Space, 61, p. 711732. 
Kovacheva M., Kostadinova-Avramova M., Jordanova N., Lanos P., Boyadzhiev Y., 2014. Extended and revised archaeomagnetic database and secular variation curves from Bulgaria for the last eight millennia. Physics of the Earth and Planetary Interiors. 236, p. 79-94.

LE Ny F., 1988. Les fours de tuiliers gallo-romains, méthodologie, étude technologique, typologique et statistique, Chronologie, Documents d'Archéologie Française, no 12, Paris.

Leonhardt R., Heunemann C., Krása D., 2004. Analyzing absolute paleointensity determinations: Acceptance criteria and the software ThellierTool4.0. Geochemistry, Geophysics, Geosystems, 5(12). doi:10.1029/2004GC000807.

NéEl L., 1955. Some theoretical aspects of rock magnetism, Phil. Mag. Suppl., 4, p. 191-243.

Pavón-Carrasco F. J., Gomez-Paccard M., Hervé G., Osete M. L., Chauvin A., 2014. Intensity of the geomagnetic field in Europe for the last $3 \mathrm{ka}$ : Influence of data quality on geomagnetic field modeling. Geochemistry Geophysics Geosystems, p. 1-16. doi:10.1002/2014GC005311.

Pavón-Carrasco F. J., Osete M. L., Torta J. M., Gaya-Piqué L. R., 2009. A regional archeomagnetic model for Europe for the last 3000 years, SCHA.DIF.3K: Applications to archeomagnetic dating. Geochemistry, Geophysics, Geosystems, 10(3), doi:10.1029/2008GC002244.

Prévot M., Mankinen E. A., Coe R. S., Grommé C. S., 1985. The Steens Mountain (Oregon) geomagnetic polarity transi- tion, 2. Field intensity variations and discussion of reversal models. Journal of Geophysical Research, 90(B12), p. 1041710448 .

Schnepp E., Lanos P., 2005. Archaeomagnetic secular variation in Germany during the past 2500 years. Geophysical Journal International, 163(2), p. 479-490.

Tema E., Hedley I., Lanos P., 2006. Archaeomagnetism in Italy: a compilation of data including new results and a preliminary Italian secular variation curve. Geophysical Journal International, 167(3), p. 1160-1171.

Tema E., Morales J., Goguitchaichvili A., Camps P., 2013. New archaeointensity data from Italy and geomagnetic field intensity variation in the Italian Peninsula. Geophysical Journal International, 193(2), p. 603-614.

Thellier E., Thellier O., 1959. Sur l'intensité du champ magnétique terrestre dans le passé historique et géologique. Annales de Géophysique, 15, p. 285-376.

Thuillier F., 2003. Les ateliers céramiques d'époque gallo-romaine dans le Nord de la Gaule. Thèse de doctorat, Tours, Université François-Rabelais, France.

Veitch R.J., Hedley I.G., Wagner J.J., 1984. An investigation of the intensity of the geomagnetic field during Roman times using magnetically anisotropic bricks and tiles. Archaeological Sciences, 37(3), Geneva, p. 359-373. 